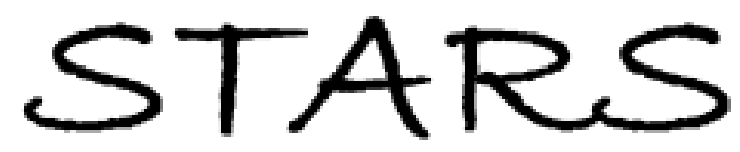

University of Central Florida

STARS

$1-1-1999$

\title{
Transverse strain responses in electrostrictive poly(vinylidene fluoride-trifluoroethylene) films and development of a dilatometer for the measurement
}

\author{
Z. -Y. Cheng \\ Vivek Bharti \\ T. -B. Xu \\ Shexi Wang \\ Q. M. Zhang
}

See next page for additional authors

Find similar works at: https://stars.library.ucf.edu/facultybib1990

University of Central Florida Libraries http://library.ucf.edu

This Article is brought to you for free and open access by the Faculty Bibliography at STARS. It has been accepted for inclusion in Faculty Bibliography 1990s by an authorized administrator of STARS. For more information, please contactSTARS@ucf.edu.

\section{Recommended Citation}

Cheng, Z. -Y.; Bharti, Vivek; Xu, T. -B.; Wang, Shexi; Zhang, Q. M.; Ramotowski, T.; Tito, F.; and Ting, R., "Transverse strain responses in electrostrictive poly(vinylidene fluoride-trifluoroethylene) films and development of a dilatometer for the measurement" (1999). Faculty Bibliography 1990s. 2581.

https://stars.library.ucf.edu/facultybib1990/2581

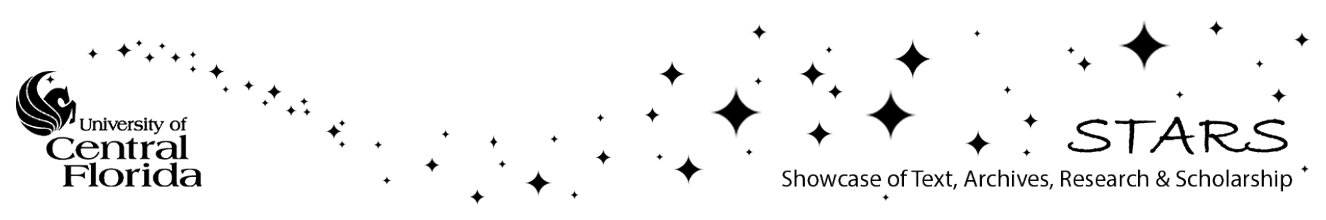




\section{Authors}

Z. -Y. Cheng, Vivek Bharti, T. -B. Xu, Shexi Wang, Q. M. Zhang, T. Ramotowski, F. Tito, and R. Ting 


\section{Transverse strain responses in electrostrictive poly(vinylidene fluoride- trifluoroethylene) films and development of a dilatometer for the measurement}

Cite as: Journal of Applied Physics 86, 2208 (1999); https://doi.org/10.1063/1.371032

Submitted: 21 January 1999. Accepted: 05 May 1999. Published Online: 29 July 1999

Z.-Y. Cheng, Vivek Bharti, T.-B. Xu, Shexi Wang, Q. M. Zhang, T. Ramotowski, F. Tito, and R. Ting

\section{ARTICLES YOU MAY BE INTERESTED IN}

Ferroelectric and electromechanical properties of poly(vinylidene-fluoride-trifluoroethylenechlorotrifluoroethylene) terpolymer

Applied Physics Letters 78, 2360 (2001); https://doi.org/10.1063/1.1358847

Transverse strain responses in the electrostrictive poly(vinylidene fluoride-trifluorethylene) copolymer

Applied Physics Letters 74, 1901 (1999); https://doi.org/10.1063/1.123707

A bimorph based dilatometer for field induced strain measurement in soft and thin free standing polymer films

Review of Scientific Instruments 69, 2480 (1998); https://doi.org/10.1063/1.1148947

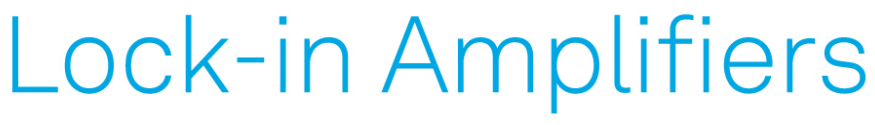
... and more, from DC to $600 \mathrm{MHz}$

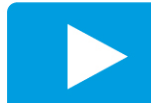

Watch 


\title{
Transverse strain responses in electrostrictive poly(vinylidene fluoride-trifluoroethylene) films and development of a dilatometer for the measurement
}

\author{
Z.-Y. Cheng, Vivek Bharti, T.-B. Xu, Shexi Wang, and Q. M. Zhang ${ }^{\text {a) }}$ \\ T. Ramotowski and F. Tito \\ Naval Undersea Warfare Center, Newport, Rhode Island 02841 \\ R. Ting \\ Chemistry Department, University of Central Florida, Orlando, Florida 32816
}

Materials Research Laboratory, The Pennsylvania State University, University Park, Pennsylvania 16802

(Received 21 January 1999; accepted for publication 5 May 1999)

\begin{abstract}
A dilatometer based on the cantilever beam concept has been developed. The dilatometer is easy to use and capable of measuring transverse strain response of soft polymer films in a broad strain range (from $10^{-7}$ to $10^{-1}$ ) without mechanical constraining of the sample. It is capable of detecting strain over a relatively wide frequency range from $\mathrm{mHz}$ to above $100 \mathrm{~Hz}$ under different load and temperature. Using the setup, the electric field induced transverse strains of the electrostrictive poly(vinylidene fluoride-trifluoroethylene) copolymer films were characterized which shows that a large transverse strain can be achieved in this class of polymer. In addition, the effect of mechanical tensile load on the transverse strain was also evaluated and the results show that the strain response will be affected by the load. However, depending on the load level, the strain response of the polymer film under a given electric field may increase or decrease with load. Based on the phenomenological theory, it is shown that for a ferroelectric based material, the mechanical load will shift the Curie temperature. Hence, to a large extent, the change of the strain response with load observed here can be understood by linking it to the strain change with temperature. (C) 1999 American Institute of Physics. [S0021-8979(99)00716-1]
\end{abstract}

\section{INTRODUCTION}

Recently, it was found that a very large longitudinal electrostrictive strain (more than $4.5 \%$ ) can be achieved in poly(vinylidene fluoride-trifluoroethylene) [P(VDF-TrFE)] copolymers under a proper electron irradiation treatment. ${ }^{1,2}$ Further more, because of the relatively high elastic modulus, the polymer also exhibits a high elastic energy density which is crucial for many electromechanical actuator and transducer applications. These features represent a significant improvement in the electromechanical performance of this new material over the conventional electroactive ceramics and polymers.

For electromechanical applications, ${ }^{3}$ in addition to the longitudinal response, the transverse response is also of great importance. In many devices operated in the longitudinal mode, a weak transverse strain response is desired which can reduce the mode coupling between the thickness direction and lateral directions for a thickness resonator or improve the device reliability. On the other hand, transverse strain responses are utilized in many areas and because the applied electric field is perpendicular to the strain direction, it offers a convenient means in generating actuation over large distances without the need to raise the driving voltage.

In this article, we report the measurement of transverse strain response in the newly developed electrostrictive

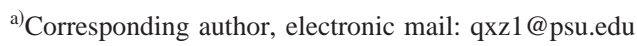

$\mathrm{P}(\mathrm{VDF}-\mathrm{TrFE})$ copolymers. In order to characterize the strain response to high fields $(\sim 50 \mathrm{MV} / \mathrm{m})$ without application of high voltages, thin polymer films of thickness about $20 \mu \mathrm{m}$ were used. One of the challenges in characterizing transverse strain response in thin polymer films is to measure the strain response in free-standing form over a broad range without introducing constraints.

In general, the strain measurement techniques can be grouped into two categories: contact methods and noncontact methods. ${ }^{4}$ The contact methods such as strain gauge and linear variable differential transformer (LVDT) which are used for transverse strain measurement in inorganic materials will impose severe constraints on polymer films due to low elastic modulus of polymers compared with inorganic materials.

For the noncontact method, although in principle laser dilatometer can be used to characterize the transverse strain response in polymer films, to reflect the laser beam mirrors are needed on two ends of the film which is a very difficult task. In addition, the sample holding is another challenge for a soft polymer film. ${ }^{5}$ Optical microscope offers a convenient means to characterize the transverse strain response. ${ }^{6}$ However, its resolution is limited so that it cannot be used at low strain response region. In the case when a high applied electric field is required, and hence, the polymer film is immersed in insulation oil to increase the breakdown voltage, the operation of an optical microscope to obtain reliable results is quite difficult. In fact, these two methods have been tested and considered for transverse strain measurement of 
newly developed polymer films and no satisfactory results can be obtained.

From both the application and fundamental understanding points of view, the electromechanical properties of an electroactive material under mechanical loads are important parameters. Thus, in developing a new technique or setup for characterizing the electromechanical behavior of materials, the capability of the setup working when the material is under mechanical loads should be an important concern.

In this article, we will first discuss the development of a new setup which is simple, convenient to use, versatile, and capable of measuring transverse strain response of polymer films over a broad strain range. The performance of setup was evaluated using the electric field induced transverse strains of both piezoelectric and electrostrictive polymeric films and the results show that the setup is capable of characterizing transverse strain responses of polymer films with a high sensitivity over a relatively wide frequency range (from near static to above $100 \mathrm{~Hz}$ ). The electric field induced transverse strains of electron irradiated (PVDF-TrFE) 65/35 mol \% copolymer films under different load conditions are also presented, which illustrates that for ferroelectric based electroactive materials, the main effect of external mechanical loads on the electromechanical responses can be understood from the shift of the ferroelectric-paraelectric transition temperature caused by the loads.

\section{DILATOMETER FOR TRANSVERSE STRAIN MEASUREMENT IN POLYMER FILMS}

The setup developed for characterizing transverse strain response of polymer films is shown schematically in Fig. 1(a). The key part of the setup is a plastic cantilever which holds the polymer film to be measured under a slight tension as shown in Fig. 1(b). As seen from the figure, the polymer film to be tested has one end clamped at a solid base (fixed) and the other end attached to the free end of the plastic cantilever which is fixed at a solid base. When the polymer film is subjected to an electric field, its expansion and contraction cause the free end of the plastic cantilever to move which can be detected using optical technique. In the current setup, a photonic sensor (Model 2000, MTI Instruments) is employed. However, if needed, a laser dilatometer can be used here to probe the movement of the free end of the cantilever which will yield a great sensitivity. This is one of the advantages of this setup, that is, the displacement probe and mechanical holding part are two separate units and can be changed separately without affecting the other part.

The photonic sensor with the optic probe (MTI 2032R) has two sensitivity ranges: the sensitivity for range 1 is 0.019 $\mu \mathrm{m} / \mathrm{mV}$ and for range 2 is $0.137 \mu \mathrm{m} / \mathrm{mV}$. All the measurements were conducted at ac mode. The output signal of the photonic sensor was measured/recorded through a lock-in amplifier (SRS Model SR 830 DSP) at small strain region and/or a digital oscilloscope (LeCroy 9310A) at high strain region of polymer films. The output signal noise of the photonic sensor, whose working frequency is from dc to 100 $\mathrm{kHz}$, is about $10 \mathrm{mV}_{p-p}$. The working frequency of the lock-in amplifier is from $0.005 \mathrm{~Hz}$ to $100 \mathrm{kHz}$. The lock-in

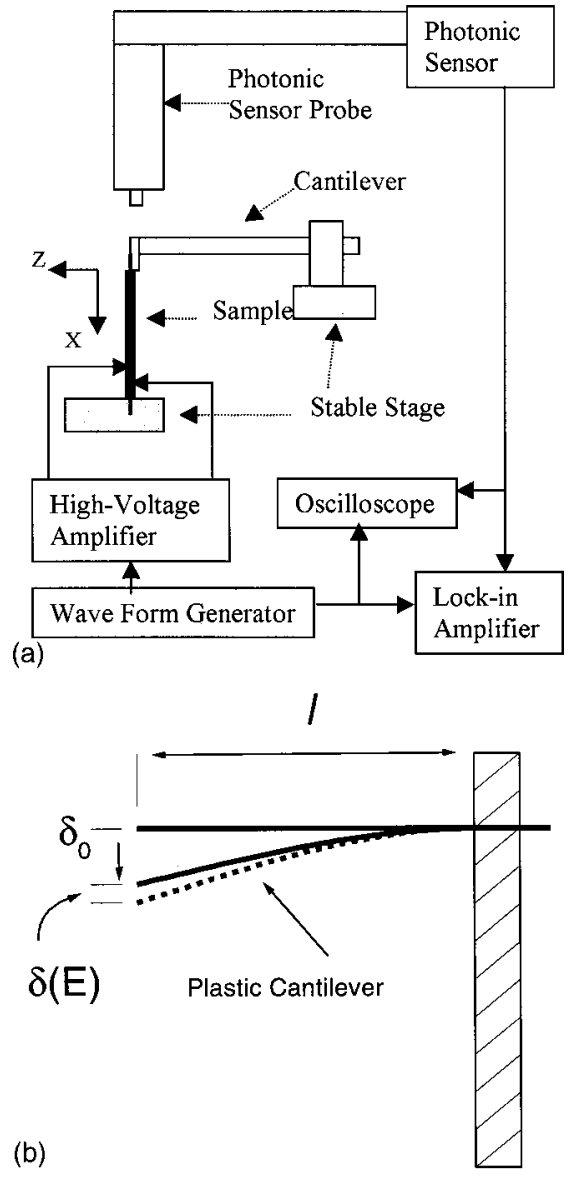

FIG. 1. Schematic of the setup (a) where the stable stage can move along $x-y-z$ directions as well as rotate about the $x$ axis. The displacement of cantilever's free end (b). In the experiment, $\delta_{0}$ and $\delta(E)$ are much smaller than $l$, the length of the cantilever

amplifier has a dynamic reserve greater than $100 \mathrm{~dB}$ which makes it possible to measure a signal of a few $\mu \mathrm{V}$ in a 10 $\mathrm{mV}$ noise background.

We now proceed to describe the details of the setup. The symbols used are summarized here. For the polymer film under test, $L$ is the length, $t$ is the thickness, $A$ is the cross section area, and $E_{s}$ is the elastic modulus in the transverse direction. The typical values of $t, A$, and $L$ are about $20 \mu \mathrm{m}$, $6 \times 10^{-2} \mathrm{~mm}^{2}$, and $12-15 \mathrm{~mm}$, respectively. For the plastic cantilever, $l$ is the length, $h$ is the thickness, $b$ is the lateral width, and $E_{c}$ is the elastic modulus. For the data presented in this section, the values of $b, h$, and $l$ are about $6,0.8$, and $80 \mathrm{~mm}$, respectively. The typical value of $\delta_{0}$, which is the static displacement of cantilever's free end from its equilibrium position as shown in Fig. 1(b), is $2 \mathrm{~mm}$. The elastic modulus of the plastic cantilever used is about $5 \mathrm{GPa}$. For the plastic cantilever with one end firmly fixed, the deflection $\delta_{0}$ at the free end will generate a static force $(F)$ which are related to each other as ${ }^{7}$

$$
\delta_{0}=\frac{1}{3} \frac{F l^{3}}{E_{C} I},
$$

where $I\left(\right.$ in $\left.^{4}\right)$ is the inertia moment of the cantilever, 


$$
I=\frac{1}{12} b h^{3} .
$$

Combing the two equations yields

$$
\delta_{0}=4 \frac{F l^{3}}{E_{C} b h^{3}}
$$

or

$$
F=\frac{E_{C} b h^{3}}{4 l^{3}} \delta_{0} .
$$

Because the polymer film is directly attached to the free end of the cantilever, this force will act as a static tensile load to the polymer film. Apparently, Eq. (3) shows that by adjusting $\delta_{0}, h$, and other parameters of the cantilever, the mechanical load can be varied over a wide range. This is another advantage of this setup.

As the polymer film is subjected to an ac electric field, its expansion and contraction will induce a corresponding ac motion in the free end of the cantilever as shown by the dashed lines in Fig. 1(b). As long as this displacement $\delta(E)$ is in the range " $\delta_{0}+\delta(E)>0$," the strain response of the film can be measured. For the piezoelectric PVDF and electrostrictive $\mathrm{P}(\mathrm{VDF}-\mathrm{TrFE})$ copolymer films studied here, $\delta(E)$ is in the range from 0 to $-0.4 \mathrm{~mm}$. As the polymer film extends or contracts under applied fields, the free end of the cantilever will move as

$$
\delta(E)=-L\left[d_{13} E+M_{13} E^{2}\right]-\frac{L}{A E_{S}} \frac{E_{C} b h^{3}}{4 l^{3}} \delta(E),
$$

where $E$ is the applied electric field (along the thickness direction, three-direction), $d_{31} E$ describes the piezoelectric effect, and $M_{13} E^{2}$ the electrostrictive effect in the polymer film, respectively. ${ }^{8}$ The last term in Eq. (4) is due to the dynamic load from the cantilever since a change in $\delta$ will cause change in the force level at the free end of the cantilever as shown in Eq. (3). In fact, this term can be made use of to evaluate the change of the elastic modulus of the polymer film with external load. The measured transverse strain response of the polymer film is

$$
\begin{aligned}
& S(E)=\frac{-\delta\left(E_{3}\right)}{L}=\frac{d_{13} E+M_{13} E^{2}}{1+\Delta}, \\
& \Delta=\frac{S_{0} L}{\delta_{0}}=\frac{E_{c} b h^{3}}{4 l^{3}} \frac{L}{A E_{S}},
\end{aligned}
$$

where, $\Delta>0, S_{0}$ is the static strain of the sample corresponding to the static load originated from the static displacement $\left(\delta_{0}\right)$ of cantilever's free end. In the current setup, $\Delta$ is much smaller than 1 (on the order of 0.001) and hence, the strain response can be linked directly to the piezoelectric and electrostrictive coefficients of the polymer evaluated, i.e.,

$$
S(E)=d_{31} E+M_{13} E^{2} .
$$

Equation (6) expresses the transverse strain response under an external electric field $E$ for a film under a constant mechanical stress. In present case, the constant stress on the sample originates from the static force which is determined by the static displacement $\left(\delta_{0}\right)$ of cantilever's free end as

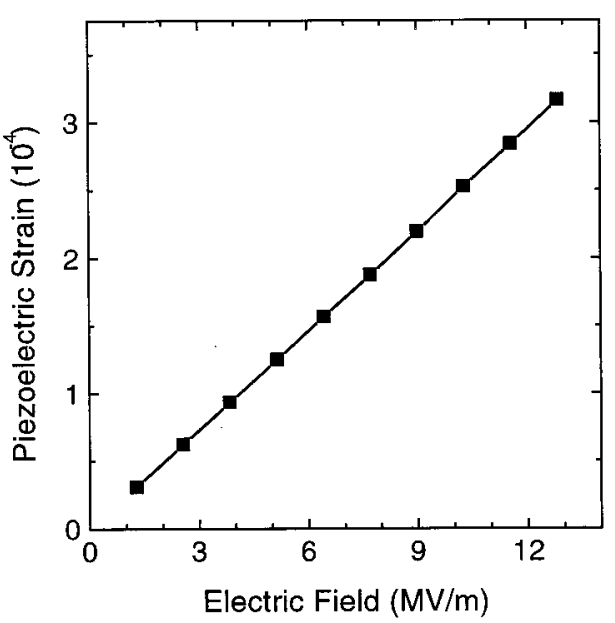

FIG. 2. Transverse strain of pure PVDF piezoelectric film under different electric fields of $1 \mathrm{~Hz}$ at room temperature.

shown in Eq. (3). Since, for most electroactive materials, both $d_{13}$ and $M_{13}$ will depend on the stress (load), ${ }^{9,10}$ the dilatometer can be used to characterize the electromechanical properties of polymeric films under different load conditions.

In the case of a sample possessing electrostrictive and piezoelectric activities, it is hard to separate the contribution from the electrostrictive and piezoelectric effects using an oscilloscope. However, one can still use a lock-in amplifier to separate and identify the contribution to the strain response from the electrostrictive and piezoelectric effects, respectively. If the applied electric field on the sample is a sine wave, the first-harmonic response of the lock-in amplifier is corresponding to the contribution from the piezoelectric effect, while the second harmonic response of the lock-in amplifier is corresponding to the contribution from the electrostrictive effect.

In the setup, special consideration and design were made so that the film under test can be immerged in an oil chamber, which serves to increase the breakdown electric field so that the measurement can be carried out to high fields. Thus, the temperature of the sample is the same as the temperature of the oil. Since the temperature of the oil can be easily changed, the setup can be used to evaluate the transverse strain responses of polymer films with temperature. During the development of the setup, it was found that in order to reduce the error in the measured strain response, it is necessary to keep the film flat. Therefore, a stage which is capable of translating the cantilever in three orthogonal directions as well as rotation in one of the axes is used.

The setup is calibrated using a commercial piezoelectric PVDF film whose $d_{31}$ value is known. As shown in Fig. 2, the strain versus applied field exhibits a linear curve, indicating the response is piezoelectric as expected. The piezoelectric coefficient $d_{13}$ is determined through the electric field strength and measured transverse strain. The results are consistent with the value from the manufacturer.

The displacement sensitivity was also evaluated using the newly developed electrostrictive P(VDF-TrFE) 65/35 mol \% copolymer and the results are presented in Fig. 3. The data were obtained at $1 \mathrm{~Hz}$ applied field with the time con- 


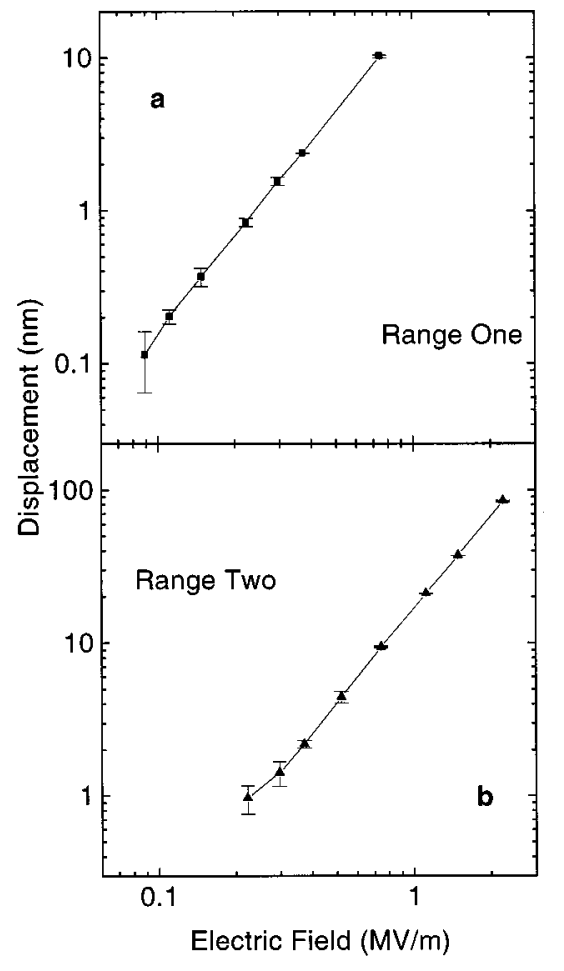

FIG. 3. Displacement sensitivity of the system when the photonic sensor is in the range one and two.

stant of $30 \mathrm{~s}$ for the lock-in amplifier. For the range 1 of the photonic sensor, the lowest measurable displacement is about $0.1 \mathrm{~nm}$, while for the range 2 , it is about $1 \mathrm{~nm}$. Considering a fact that $L \approx 10 \mathrm{~mm}$, the results indicate that the setup is capable of measuring transverse strain to $10^{-7}$ range. On the other hand, the photonic sensor used here can measure displacement up to $0.7 \mathrm{~mm}$ range which corresponds to a strain in polymer films of 0.07 . However, photonic sensors with the upper range to more than $5 \mathrm{~mm}$ is commercially available. Clearly, the separation of the sample holding unit and displacement sensing unit in the set up enables us to characterize the strain in polymer films over a very wide range. In addition, the linear $\log -\log$ plot of the data in Fig. 3 implies that the strain response of the polymer follows $S(E)=M_{13} E^{n}$ relation (power law) and slopes yield $n=1.99$ and $n=2.08$, respectively, which is consistent with the longitudinal strain data on the similar films measured in the low field range $(<10 \mathrm{MV} / \mathrm{m})$ and shows that the response is electrostrictive $(n=2) .{ }^{2}$ The electrostrictive coefficient $M_{13}$ calculated from the data in Fig. 3 is 1.9 $\times 10^{-18} \mathrm{~m}^{2} / \mathrm{V}^{2}$.

The operation frequency range is another concern when developing a strain measurement set up. At the low frequency end, the frequency limit depends on the frequency range of a lock-in amplifier and the strain level of the sample. The limiting frequency for the former is $0.005 \mathrm{~Hz}$ in the present system. And the later is caused by the increased noise of the environment which has approximately $1 / f$ spectrum, where $f$ is frequency. On the high frequency end, the operation frequency is limited by the resonance frequency $\left(f_{1}\right)$ of the cantilever. The lowest resonance frequency of a cantilever with one end fixed and the other free is given by ${ }^{11}$
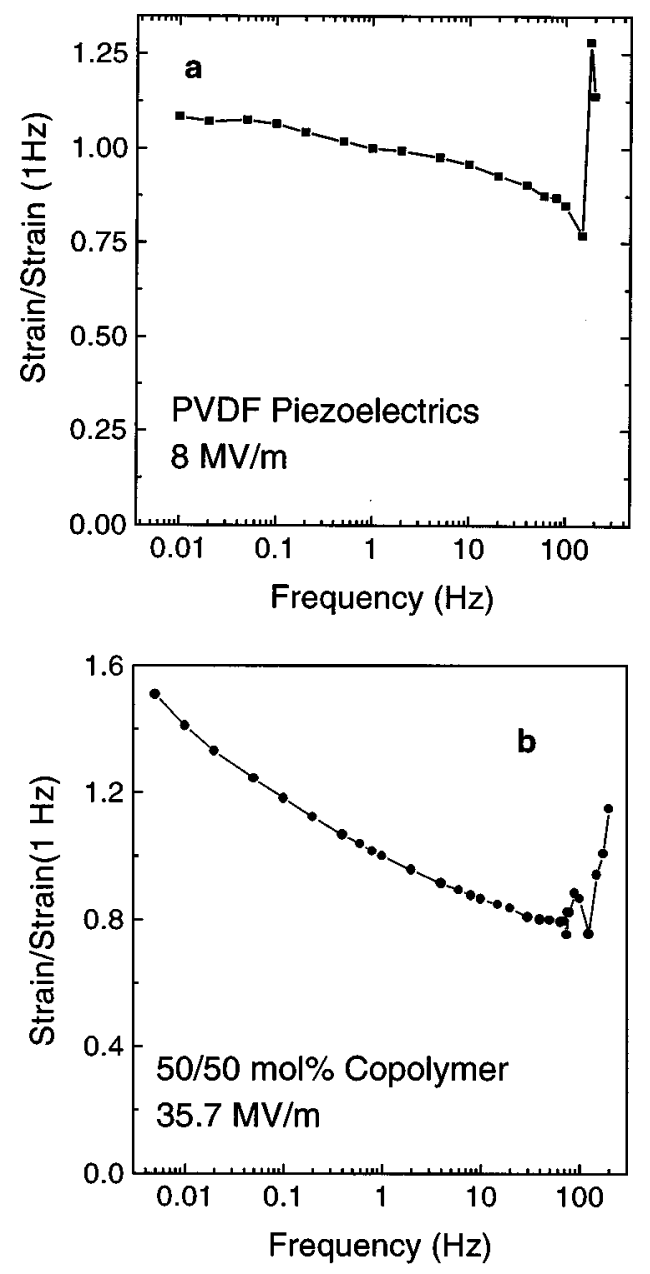

FIG. 4. Frequency dependence of the electric field induced transverse strain of a PVDF piezoelectric film and (b) $\mathrm{P}(\mathrm{VDF}-\mathrm{TrFE})$ electrostrictive film under a constant electric field.

$$
f_{1}=0.16154 \frac{h}{l^{2}} \sqrt{\frac{E_{C}}{\rho}}
$$

while the lowest resonance frequency of a cantilever with two ends fixed is determined by ${ }^{11}$

$$
f_{1}^{\prime}=1.0279 \frac{h}{l^{2}} \sqrt{\frac{E_{C}}{\rho}}
$$

where $\rho$ (in $\mathrm{kg} / \mathrm{m}^{3}$ ) is the density of the plastic probe used. Since in our setup, one end of the cantilever is fixed firmly at a base and another end is not totally free (attached to the polymer film), the resonance frequency should be somehow in between the $f_{1}$ and $f_{1}^{\prime}$ of Eq. (7).

Therefore, as the polymer film is driven electrically with increasing frequency, a series of resonance in the cantilever will be excited. For example, the frequency response data using the commercial piezoelectric PVDF film as the polymer sample attached to the cantilever probe is presented in Fig. 4(a). A resonance was observed at a frequency of the ac driving electric field at about $170 \mathrm{~Hz}$ for a cantilever beam with $\rho=1.47 \times 10^{3} \mathrm{~kg} / \mathrm{m}^{3}$ and $E_{c}=5 \mathrm{GPa}$, and the result is in accord with what was expected from Eq. (7). The weak frequency dependence of the piezoelectric coefficient at frequencies below $50 \mathrm{~Hz}$ as shown in Fig. 4(a) is consistent 


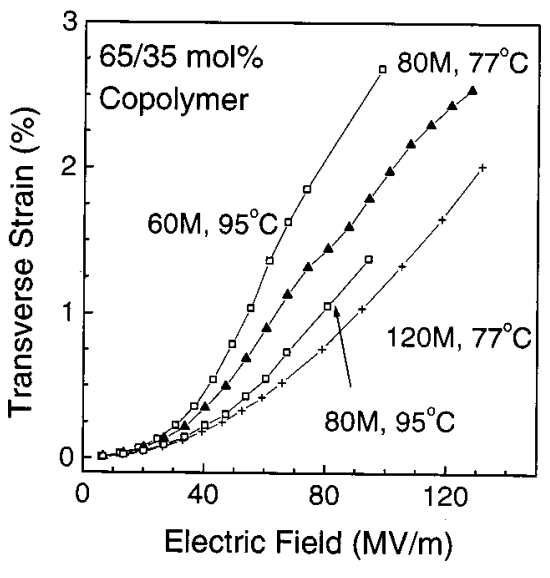

FIG. 5. Electric field dependence of the transverse strain measured at room temperature for stretched $65 / 35 \mathrm{~mol} \% \mathrm{P}(\mathrm{VDF}-\mathrm{TrFE})$ copolymer films under different electron irradiation conditions.

with what measured in a thick copolymer sample (mm thickness) of $\mathrm{P}$ (VDF-TrFE) $75 / 25 \mathrm{~mol} \%$ piezoelectric copolymer. ${ }^{5}$ With the same probe, the frequency dependence of transverse strain response of a newly developed electrostrictive copolymer film under a field of $35.7 \mathrm{MV} / \mathrm{m}$ [P(VDFTrFE) $50 / 50 \mathrm{~mol} \%$ irradiated at $95^{\circ} \mathrm{C}$ with $100 \mathrm{Mrad}$ dose] was also characterized. As shown in Fig. 4(b), there is a resonance at a frequency near $90 \mathrm{~Hz}$ of the ac driving electric field. The apparent lowering of the resonance frequency here is caused by the electrostrictive response of the polymer film $\left(S=M E^{2}\right)$ which generates a mechanical motion of $2 f$ frequency when the driving electric field has a frequency $f$. Apparently, at below $50 \mathrm{~Hz}$, the effect of the resonance to the measured data is negligible here. By adjusting the dimension of the cantilever probe, one can raise the resonance frequency to above $500 \mathrm{~Hz}$ which makes it possible to characterize the transverse strain response of a polymer film up to more than $100 \mathrm{~Hz}$ if needed.

\section{TRANSVERSE STRAIN RESPONSE OF IRRADIATED P(VDF-TRFE) 65/35 COPOLYMER}

In this section, the change of the transverse strain of stretched films ( $5 \times$ stretching ratio) of $\mathrm{P}(\mathrm{VDF}-\mathrm{TrFE}) 65 / 35$ copolymer with mechanical load was evaluated. Shown in Fig. 5 is the transverse strain response as a function of applied field measured at room temperature for films with different irradiation conditions under load free condition. Clearly, the response depends strongly on the irradiation condition.

With regard to electroactive materials for electromechanical applications, although the electric field induced strain response at load free condition is important to understand and to characterize the materials behavior, the material response when subjected to external load is crucial for most of the device applications. Needless to say, the information is also valuable from a basic understanding point of view. Here, the film irradiated at $95^{\circ} \mathrm{C}$ with $60 \mathrm{Mrad}$ dosage is examined under different mechanical load conditions, since this is the one that exhibits the best transverse strain response at load free condition as seen from Fig. 5. In order to vary
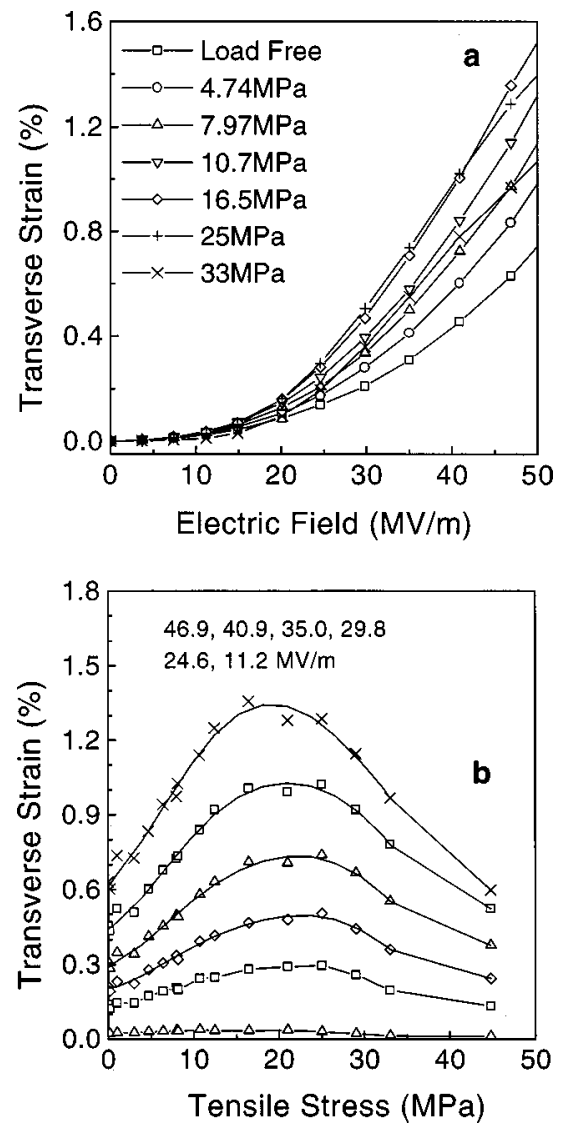

FIG. 6. Load effect on the electric field induced transverse strain response measured at room temperature with applied electric field of $1 \mathrm{~Hz}$ for $65 / 35$ mol \% P(VDF-TrFE) copolymer film irradiated at $95^{\circ} \mathrm{C}$ with $60 \mathrm{Mrad}$ dosage (a). The relation between the strain response and electric field for films under different loads (b). The relation between strain response and static load for films at different electric field strengths. Symbols in figure express the measured data, while solid lines in figure are drawn to guide eyes.

the mechanical load over a broad range, plastic cantilever beams with thickness from 0.8 to $4 \mathrm{~mm}$ were used. The $E_{c}$ of the plastic trips is from 2.5 to $6 \mathrm{GPa}$. In addition, the static displacement $\left(\delta_{0}\right)$ of cantilever's free end was varied from 2 to $6 \mathrm{~mm}$. Thus, the static tensile load on the polymer film can change from nearly zero to more than $45 \mathrm{MPa}$.

The relation between the electric field and the transverse strain response for the sample under different static tensions is shown in Fig. 6(a). The static tension dependence of electric field induced strain for the same material at different external electric fields is shown in Fig. 6(b). In these experiments, the load (stress) was along the stretching direction of the copolymer film and the transverse strain was also measured along the stretching direction. Although the strain response of a sample at the same electric field strongly depends on the static tension, it is found that the strain response for the sample under different static load conditions still linearly depends on the square of the electric field strength. This is what is expected from the electrostrictive effect. ${ }^{1,2}$ However, the electrostrictive coefficient depends strongly on the static load.

The results in Fig. 6 indicate that for a given field, the transverse strain increases with load initially and then decreases with the load after reaching the maximum. For the 
polymer studied here, the electric field induced strain response mainly originates from the phase changes from a nonpolar to a polar phase under external electric field., ${ }^{12,13}$ Hence, this load dependence behavior can be understood based on how this change is affected by the load.

Before the electron irradiation, the copolymer exhibits typical first-order phase transition from para- to ferro-electric phase. ${ }^{1,14}$ However, after the electron irradiation, the physical properties of the copolymer are very similar to that of relaxor ferroelectrics. For example, the dielectric constant maximum temperature $\left(T_{m}\right)$ and dielectric loss maximum temperature $\left(T_{m}^{\prime}\right)$ are strongly dependent on the frequency; and at the same frequency $T_{m}$ is higher than $T_{m}^{\prime} .{ }^{1}$ These are the basic features of dielectric relation phenomena observed in relaxor ferroelectrics. ${ }^{15}$ In addition, it was found that the relation between $T_{m}$ and frequency for these copolymers follows well the Vogel-Fulcher law. ${ }^{1,16}$ The Vogel-Fucher law was widely used in relaxor ferroelectrics to express the relation between $T_{m}$ and frequency. ${ }^{17}$ More importantly, it was found that these copolymers exhibit a slim polarization hysteresis loop, that the remanent polarization gradually increase with decreasing temperature, and that the ferroelectric state can be observed at low temperature. ${ }^{1,17}$ These are exactly the polarization behaviors observed in relaxor ferroelectrics. ${ }^{18}$ With regard to the ferroelectric behavior of the relaxor ferroelectrics, it is found that the depolarization temperature $\left(T_{d}\right)$ is generally lower than $T_{m}$ by a few tens degrees. ${ }^{19}$ It was found that $T_{d}$ for the copolymer studied here is about $40-60{ }^{\circ} \mathrm{C}$ lower than $T_{m} \cdot{ }^{16}$

For relaxor ferroelectrics, it is known that the local phase transition temperature (or the freezing temperature of the polar region) is distributed in a very broad range and that the density of the frozen polar regions increases with decreasing temperature. ${ }^{18,19}$ In addition, the volume density of the frozen polar regions increases with external electric field, which is also called the electric field induced phase transition. ${ }^{19}$ That is why the large electrostrictive coefficient was obtained in relaxor ferroelectrics. ${ }^{20}$ Therefore, the dielectric behavior and the electric field induced strain response strongly depends on the temperature. It is believed that the density of the frozen polar region is relatively higher and the interaction between the frozen polar regions is still not high at temperatures near $T_{m} \cdot{ }^{19}$ However, at temperatures lower than $T_{d}$, most of the polar regions are frozen and the interaction among the frozen polar regions is relatively high. Thus, both $T_{m}$ and $T_{d}$ can be used as parameters to characterize the average phase transition temperature of local transition between para- and ferroelectric phases. For the material studied here, the $T_{m}$ is about $27^{\circ} \mathrm{C}$ at $30 \mathrm{~Hz}$ as measured from the dielectric data. ${ }^{21}$ It has been observed that for the relaxor ferroelectrics under a relatively high driving electric field, the induced strain increases as the temperature is lowered towards $T_{d} \cdot{ }^{22}$ However, as the temperature is lowered further, the strain response will decrease due to increased $180^{\circ}$ domain wall motions associated with the macropolarization switching.

Based on Devonshire phenomenological theory, for a ferroelectric material under a stress $X_{i}$ and with a polarization $\left(P_{3}\right)$ along ' 3 "' direction, if only consider the contribu- tion to the free energy of the system from the electrostrictive effect (first order approximate), ${ }^{23}$ the shift of phase transition temperature with stress is

$$
\Delta T=2 \varepsilon_{0} C Q_{i 3} X_{i},
$$

where $\Delta T=T_{c}\left(X_{j}\right)-T_{c}(0), \varepsilon_{0}=8.85 \times 10^{-12} \mathrm{~F} / \mathrm{m}, C$ is the Curie-Weiss constant, $Q_{i 3}$ is the electrostrictive coefficients defined as $S_{i}=Q_{i 3} P_{3}^{2}$, and $T_{c}$ is the Curie temperature. Although the Eq. (8) is written for a single-crystal normal ferroelectrics, the trend should be the same also for the relaxor ferroelectrics considering the microstructure of the relaxor ferroelectrics. For relaxor ferroelectrics, the stress will shift both $T_{m}$ and $T_{d}$ and the direction of the shifting will depend on the sign of $Q$, the electrostrictive coefficient. For the polymer investigated here, $Q_{13}>0$ and the applied stress is $X_{1}>0$. Thus, Eq. (8) shows that $\Delta T>0$. That is, both $T_{m}$ and $T_{d}$ of the sample will move to a higher temperature with the tensile load.

This result indicates that the measured strain responses at room temperature for films under different load are, to a large extent, equivalent to the strain responses measured at different temperatures under stress free condition. Since $T_{m}$ for the polymer under free stress condition is close to room temperature, the measured strain response will increase with tensile stress. This is what was obtained at the low load range as shown in Fig. 6. However, as the load increases further which moves $T_{d}$ to higher than room temperature, the strain response will decrease. Below $T_{d}$, most of polar regions are frozen and the interaction among the frozen polar regions is very high. Thus, the contribution to the electric field induced strain from the para- to ferro-electric phase transition decreases. This is why the electrostrictive response decreases with load in the high load region as shown in Fig. 6.

\section{SUMMARY}

A new dilatometer is developed for characterizing the electric field induced transverse strain of free-standing polymer films under different load conditions and at different temperatures. The setup is easy to use, can be operated in a broad strain range with high sensitivity, and has a reasonable operation frequency range, from $\mathrm{mHz}$ to above $100 \mathrm{~Hz}$. Using the dilatometer, the electric field induced transverse strains of high energy electron irradiated P(VDF-TrFE) films were characterized. It was found that a high transverse strain response can be achieved in this class of material which depends crucially on the irradiation condition. It was also found that the field induced strain will change with external load. For example, for P(VDF-TrFE) 65/35 mol \% copolymer irradiated at $95^{\circ} \mathrm{C}$ with $60 \mathrm{Mrad}$ dosage, the transverse strain measured at room temperature increases with tensile load initially until a load near $15 \mathrm{MPa}$, beyond that the strain decreases slowly with the load. Based on Devonshire phenomenological theory, it can be shown that for the polymer investigated here, the tensile stress will favor the low temperature phase and the change of the strain with load can be understood qualitatively from the shifting of $T_{m}$ and $T_{d}$ due to the load. In general, for ferroelectric based materials, the 
dependence of strain response with load can be understood from the shifting of the Curie temperature with stress.

\section{ACKNOWLEDGMENTS}

This work was supported by the National Science Foundation through Grant No ECS-9710459 and the Office of Naval Research through Grant No N00014-97-1-0900.

${ }^{1}$ Q. M. Zhang, V. Bharti, and X. Zhao, Science 280, 2101 (1998).

${ }^{2}$ X. Zhao, V. Bharti, Q. M. Zhang, T. Romotowski, F. Tito, and R. Ting, Appl. Phys. Lett. 73, 2054 (1998).

${ }^{3}$ J. M. Herbert, Ferroelectric Transducers and Sensors (Gordon and Breach, New York, 1984).

${ }^{4}$ J. Su, P. Moses, and Q. M. Zhang, Rev. Sci. Instrum. 69, 2480 (1998).

${ }^{5}$ H. Wang, Ph.D. thesis, The Pennsylvania State University, 1994.

${ }^{6}$ R. E. Pelrine, R. D. Kornbluh, and J. P. Joseph, Sens. Actuators A 64, 77 (1998).

${ }^{7}$ K. Lingaiah, Machine Design Data Handbook (McGraw-Hill, New York, 1994).

${ }^{8}$ IRE Standards on Piezoelectric Crystals, Proc. IRE 49, 1169 (1961).

${ }^{9}$ Q. M. Zhang, W. Y. Pan, S. J. Jang, and L. E. Cross, Ferroelectrics 88, 147 (1988).
${ }^{10}$ J. Zhao et al., Appl. Phys. Lett. (to be published).

${ }^{11} \mathrm{~J}$. Merhaut, Theory of Electroacoustics (McGraw-Hill, New York, 1981).

${ }^{12}$ Z.-Y. Cheng, T.-B. Xu, V. Bharti, S. Wang, and Q. M. Zhang, Appl. Phys. Lett. 74, 1901 (1999)

${ }^{13} \mathrm{~K}$. Tashiro, in Ferroelectric Polymers, edited by H. S. Nalwa (Dekker, New York, 1995), pp. 63-181.

${ }^{14}$ T. Furukawa, Phase Transit. 18, 143 (1989).

${ }^{15}$ Z.-Y. Cheng, R. S. Katiyar, X. Yao, and A. Guo, Phys. Rev. B 55, 8165 (1997).

${ }^{16}$ V. Bharti, Z.-X, Zhao, Q. M. Zhang, T. Romotowski, F. Tito, and R. Ting, Mater. Res. Innovat 2, 57 (1998).

${ }^{17}$ D. Viehland, S. J. Jang, L. E. Cross, and M. Wutting, J. Appl. Phys. 68, 2916 (1990).

${ }^{18}$ L. E. Cross, Ferroelectrics 76, 241 (1987).

${ }^{19}$ Z.-Y. Cheng, R. S. Katiyar, X. Yao, and A. S. Bhalla, Phys. Rev. B 57, 8166 (1998).

${ }^{20}$ K. Uchino and S. Nomura, J. Mater. Sci. 16, 569 (1981).

${ }^{21}$ Q. M. Zhang (unpublished).

${ }^{22}$ J. Zhao, Q. M. Zhang, N. Kim, and T. Shrout, Jpn. J. Appl. Phys., Part 1 34, 5658 (1995).

${ }^{23}$ M. E. Lines and A. M. Glass, Principles and Applications of Ferroelectrics and Related Materials (Oxford University Press, New York, 1977). 